\title{
Protein Adsorption at Interfaces Detected by Second Harmonic Generation
}

\author{
J. S. Salafsky and K. B. Eisenthal* \\ Department of Chemistry, Columbia University, 3000 Broadway, Mail Code 3107, New York, New York 10027
}

Received: April 4, 2000; In Final Form: June 9, 2000

\begin{abstract}
We show that second harmonic ( $\mathrm{SH}$ ) spectroscopy, an intrinsically surface-selective technique, can be used to monitor protein (cytochrome $c$ ) adsorption to silica surfaces and negatively charged supported phospholipid bilayers. The origin of the SH signal is due to the effect of the adsorbed protein on the water molecules polarized near the charged interface. Although the protein does not contribute its own SH signal, its binding to the glass surface or the membrane reduces the polarization of the interfacial water molecules and this effect is proportional to the adsorbed protein concentration and can be monitored with high precision, in real time. The free energy of adsorption $\left(\Delta G_{\text {ads }}=-11.8 \mathrm{kcal} / \mathrm{mol}\right)$ of cytochrome $c$ to glass was determined from an adsorption isotherm measurement of the SH signal as a function of the bulk protein concentration. A detection sensitivity of $0.1 \mathrm{pmol} / \mathrm{cm}^{2}$ of adsorbed cytochrome $c$ protein is readily achieved.
\end{abstract}

\section{Introduction}

Interfacial binding or adsorption of proteins to surfaces and membranes is an important process in cell biology, ${ }_{1}^{1}$ studies involving biomembranes, ${ }^{2,3}$ and biosensor devices. ${ }^{4}$ Detection of proteins at interfaces must often be accomplished by labeling the protein with a fluorescent or radioactive tag. Surface plasmon resonance (SPR) offers a means of detecting unlabeled proteins on the basis of their mass altering the dielectric constant at the interface, but it requires a metal film for generation of the plasmon wave, ${ }^{5,6}$ as do electrical methods of detection, and this may complicate the measurement if the protein denatures at the metal surface. A metal surface such as gold may also be incompatible with supported lipid bilayers which are useful as model membranes for studying protein-cell or protein-protein interactions. ${ }^{7,8}$ Here we present a method based on second harmonic generation for detection of proteins adsorbed to interfaces and in the presence of bulk species. The method is significantly more sensitive than surface plasmon resonance or circular dichroism in its ability to detect single or submonolayers of protein and requires no labels. In addition, the technique requires no metal layer and so is fully compatible with supported lipid bilayers, and it works with nonplanar interfaces such as bead surfaces or liposomes - unlike SPR which requires a planar interface. As a first demonstration of the technique, we focus on cytochrome $c$ and myoglobin adsorption to silica surfaces and to supported lipid bilayers.

\section{Theoretical Background}

Second harmonic ( $\mathrm{SH}$ ) spectroscopy is a nonlinear, surfaceselective technique for detecting molecules within a molecularly thin layer near a surface..$^{9,10}$ The molecules are oriented by the surface through chemical or electrostatic forces and irradiation with a fundamental beam $(\omega)$ leads to the generation of second harmonic $(2 \omega)$ light; molecules in the bulk, which are randomly oriented, produce no SH light. Generally, SH studies of molecules involve species with a large molecular hyperpolarizability $(\beta)$ detected in a resonantly enhanced process. However,

* Author to whom correspondence should be addressed. it has been shown that water at a charged interface, silica for example, produces an SH signal, due to both the monomolecular water layer oriented directly at the interface and a longer-range contribution due to the water molecules becoming polarized by the static electric field of the charged surface. ${ }^{11,12}$

Direct detection of proteins at interfaces using SHG has been done only with bacteriorhodopsin purple membranes. ${ }^{13}$ The requirements for $\mathrm{SH}$ production, namely, net orientation of a noncentrosymmetric (SHG-active) molecule at the interface, are difficult to satisfy with proteins. Possible sources of SH light generated by the protein are the amino acids with aromatic side chains, such as tryptophan and tyrosine, which absorb in the near-UV region around $280 \mathrm{~nm}$. However, these may be randomly distributed throughout the protein and the protein itself non-oriented at the interface, greatly reducing the number which could contribute to the SH signal. Yet another possibility for directly measuring protein SH light arises in the case of proteins with cofactors, such as the porphyrin heme group in cytochrome $c$. However, these macrocycles are usually centrosymmetric, or nearly so, and are not expected to be SHG-active. The SH process is also coherent and thus depends quadratically on the number density of $\mathrm{SH}$-active molecules making the requirements for $\mathrm{SH}$ detection even more stringent.

In this paper we show that, alternatively, the SH signal due to the water polarized by the charged surface can be used to detect adsorption of charged protein molecules at silica and negatively charged supported lipid bilayers. The production of SH light by water at a charged interface can be described by the following equation:

$$
\sqrt{I_{\mathrm{SH}}} \propto E_{2 \omega}=A \chi^{2}+B \Phi_{0} \chi^{3}
$$

where $I_{\mathrm{SH}}$ is the $\mathrm{SH}$ intensity, $E$ is the electric field of the $\mathrm{SH}$ light, $\chi^{2}$ and $\chi^{3}$ are the second- and third-order nonlinear susceptibilities, $\Phi$ is the surface potential, and $A$ and $B$ are constants which depend on the specific properties of the surface. ${ }^{12}$ In effect, the total $\mathrm{SH}$ light generated is due to both a monomolecular surface contribution $\left(\chi^{2}\right.$ part) and that due to the polarization of water molecules by the electric field of the charged surface $\left(\chi^{3}\right.$ part). The electric potential of a charged 


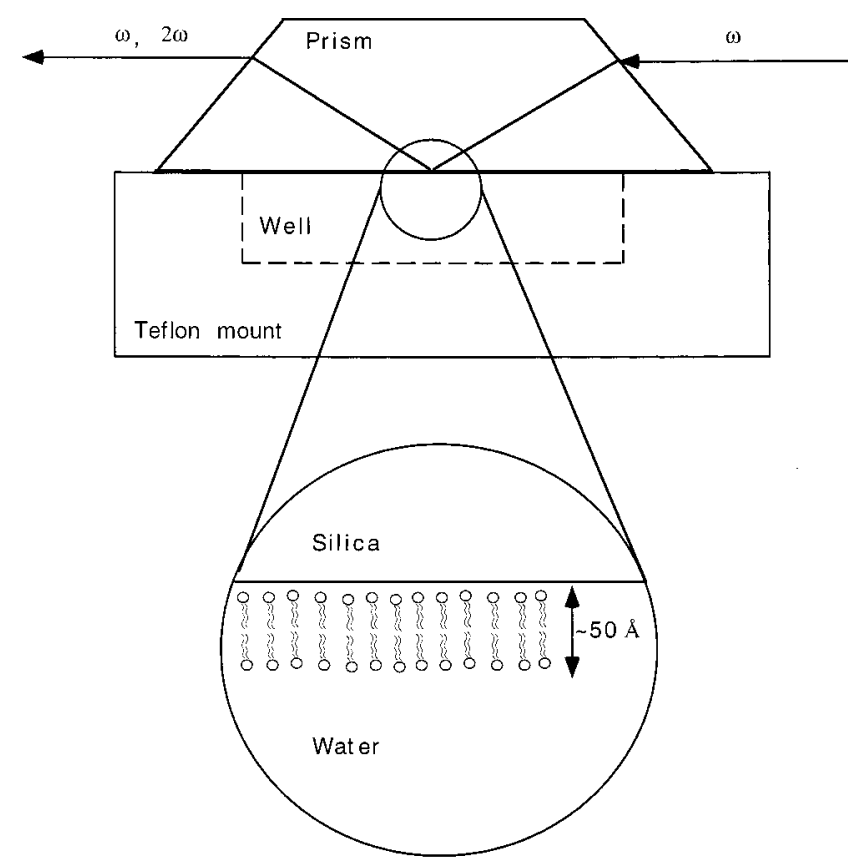

Figure 1. Schematic of the prism and geometry used in our experiments. The fundamental light $(\omega)$ undergoes total internal reflection at the silica or silica-lipid bilayer interface to produce the second harmonic beam $(2 \omega)$. Shown here is the supported lipid bilayer on the silica surface. Experiments were performed with both the silica alone and with a supported lipid bilayer membrane.

surface in contact with electrolyte, and its decay to the bulk value follows the well-known Gouy-Chapman model where

$$
\sigma=11.7 \sqrt{C} \sinh \left(19.5 z \Phi_{0}\right)
$$

with $\sigma$ the surface charge density $\left(\mu C / \mathrm{cm}^{2}\right), C$ the bulk electrolyte concentration, $z$ the charge of the electrolyte, $\Phi_{0}$ the surface potential, and $\Phi$ the potential at a distance $x$ from the surface. At $\mathrm{pH} 7$, the number density of negative surface charges for both the silica and the supported lipid bilayers used in our experiments (4:1 DOPC:DOPG) is expected to be on the order of $10^{13} / \mathrm{cm}^{2}{ }^{12,15}$ The decay of the surface potential to the bulk value is dependent on the surface potential and Debye length, $\kappa$, and occurs over a distance of several to hundreds of nanometers, depending on the bulk electrolyte concentration.

\section{Experimental Methods}

For our experiments, we used a silica prism and total internal reflection (TIR) geometry as shown in Figure 1. The experimental setup consists of a Tsunami mode-locked laser (femtosecond pulses, $1 \mathrm{~W}$ average power, $82 \mathrm{MHz}$ repetition rate) and single-photon detection electronics and for the sample, a clean fused silica prism was mounted in a Teflon holder and suspended over a well containing water, buffer, or a protein solution. Briefly, the beam of an argon ion laser $(10.5 \mathrm{~W})$ is directed into the cavity of a titanium sapphire mode-locking laser (Tsunami). The output serves as the fundamental $(\omega)$ and occurs at a repetition rate of $82 \mathrm{MHz}$ with $\sim 150$ fs pulse duration $(800-834 \mathrm{~nm})$ and $1.1-1.3 \mathrm{~mW}$ power. For all experiments, the polarization of the fundamental was set to $-45^{\circ}$ from the normal of the laser table. The fundamental beam was directed on to one face of the Dove prism where it is refracted and totally internally reflects at the glass-water interface, as shown in Figure 1. Second harmonic light is generated within an evanescent wavelength $(\sim 100 \mathrm{~nm})$ from the silica surface.
Detection of the second harmonic photons at $2 \omega$ was accomplished using a series of focusing lenses, a monochromator, and a photomultiplier tube in single photon-counting mode.

Liposomes were prepared by the freeze-thaw method using a mixture of phospholipids (4:1 DOPC:DOPG) (1,2-dioleoylsn-glycero-3-[phospho-rac-1-glycerol] and 1,2-dioleoyl-sn-glycero-3-phosphocholine) doped with $1 \mathrm{~mol} \%$ of a fluorescent dye-lipid-Texas Red 1,2-dihexadecanoyl-sn-glycero-3-phosphoethanolamine triethylammonium salt (Texas Red, DHPE; Molecular Probes, Inc.), as described previously. ${ }^{8}$ The addition of $25 \mathrm{~mol} \mathrm{\%}$ of the negatively charged lipid (DOPG) corresponds to a bilayer surface charge density of $3.5 \times 10^{5}$ charges/ $\mathrm{cm}^{2}$ using an average molecular area per lipid of $70 \AA^{2}$. Phospholipid bilayers were formed from these on the underside of a glass Dove prism (type BK-7; Melles Griot) mounted in a piece of Teflon and suspended over a well, by filling the well with the liposome solution and rinsing out after several minutes as described in ref 8 . Fluorescence recovery after photobleaching (FRAP) experiments were performed using a Biorad MRC-600 confocal fluorescence microscope with appropriate filters for the Texas Red dye, and focused on the membrane plane; these experiments were performed on supported bilayers prepared on glass coverslips as a check for membrane integrity: the supported bilayers are uniform and the lipid molecules fluid as demonstrated by the recovery of the dye-lipid fluorescence following photobleaching within several minutes. The prism was previously cleaned by soaking for $45 \mathrm{~min}$ at $90{ }^{\circ} \mathrm{C}$ in a mixture of freshly prepared 30:70 $\mathrm{H}_{2} \mathrm{O}_{2}: \mathrm{H}_{2} \mathrm{SO}_{4}$ (by vol., "Piranha solution"). The well underneath the prism, with or without the supported bilayer present, was filled with double-distilled deionized water ( $\mathrm{pH} 7$ ) and the underside of the prism was never exposed to air after formation of the supported bilayer.

Cytochrome $c$ (horse heart; Sigma) is a globular protein with $\mathrm{a} \sim 15 \AA$ radius and $13 \mathrm{kDa}$ molecular weight and contains a single heme cofactor. The protein is positively charged (+9) at neutral $\mathrm{pH}(\mathrm{pI}=10.5)$, and has been shown to bind to negatively charged surfaces such as lipid bilayer membranes ${ }^{16,17}$ and silica. $^{18}$

\section{Results}

The SH signal of the silica or silica-membrane interface in distilled, deionized water or $10 \mathrm{mM}$ phosphate buffer $(\mathrm{pH} \mathrm{7})$ was measured (the signal is on the order of $10^{4}$ counts per second); as protein aliquots are added to the well underneath the prism, the signal decreases, eventually reaching an asymptotic value. A typical spectrum of the $\mathrm{SH}$ signal for the silica interface alone and in the presence of protein is shown in Figure $2 \mathrm{a}$. The kinetics of this process was monitored in time and shows an immediate decrease in the signal (Figure 2b) within the response time of our setup $(\sim 1 \mathrm{~s})$. A saturating amount of cytochrome $c(5 \mu \mathrm{M})$ reduces the $\mathrm{SH}$ signal by a factor of about 2 for water and slightly less in the presence of $10 \mathrm{mM}$ phosphate buffer, due to its higher electrolyte concentration. To check that the reduction of the signal was not due to local heating effects, the signal was measured to be the same after the fundamental beam $(\omega)$ had been blocked for up to $20 \mathrm{~min}$. To find out whether the cytochrome $c$ contributes its own SH signal through a resonantly enhanced $\chi^{2}$ process, the fundamental wavelength was tuned across the absorption band of the cytochrome (Figure 2 inset). The measured SH signal remained essentially constant to within $15 \%$ while the absorption changed by a factor of 6 , indicating that the reduction in the $\mathrm{SH}$ signal was not due to absorption of the $\mathrm{SH}$ light or two-photon absorption of the fundamental by the protein. We could also detect cytochrome 

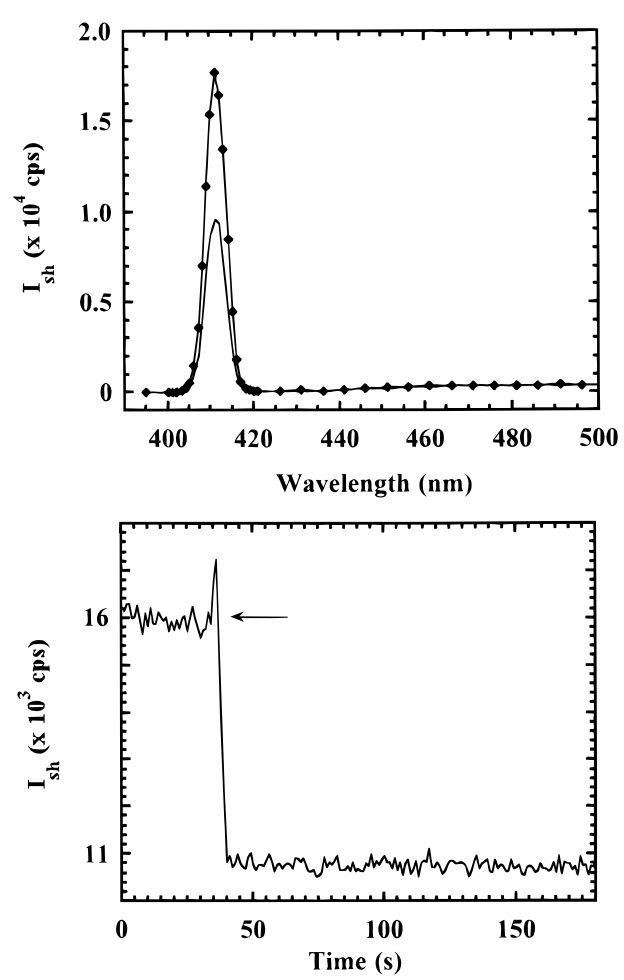

Figure 2. (a) SHG spectrum of silica-water interface ( $)$ and in the presence of $2 \mu \mathrm{M}$ cyt $c$ showing a reduction of the SH intensity with cyt c. The peak of the spectrum occurs at $412 \mathrm{~nm}(\omega=824 \mathrm{~nm})$. The full width at half-maximum of the spectrum is determined by the spectral width of the fundamental light. (b) Cytochrome $c$ absorption spectrum in aqueous solution. The $(\$)$ symbols indicate the $2 \omega$ wavelengths the fundamental beam was tuned to. No significant change in the SH intensity was detected over this spectral range (407-442 $\mathrm{nm}$ ) while the absorbance changes by a factor of more than 6 , indicating that the cytochrome absorption plays no role in the $\mathrm{SH}$ reduction. Kinetics trace after the addition of a less than saturating amount of cyt $c$. The signal drop is immediate and remains constant in time.

$c$ binding to lipid bilayer membranes, a well-known phenomenon: a supported, negatively charged lipid bilayer (4:1 DOPC: DOPG) was prepared on the prism surface and a similar reduction was found in the $\mathrm{SH}$ signal with cyt $c$, although the magnitude of the SH signal was slightly smaller both for the background and in the presence of the protein.

Several possibilities might account for the reduction of the $\mathrm{SH}$ signal in the presence of cyt $c$. First, the protein sterically reduces the amount of water near the interface, thus reducing the volume of polarized water within the evanescent wavelength of the totally internally reflected light. However, in our experiments with deionized water the decay of the surface potential is expected from Gouy-Chapman theory to occur over a distance of tens of nanometers, even allowing for the ionic strength of the added protein. The adsorbed cyt $c$ would thus occupy only $1-2 \mathrm{~nm}$ of the optical region determined by the evanescent wave $(\sim 100 \mathrm{~nm})$ and thus the SH signal would be unaffected by the adsorbed protein. Another more likely possiblity is that the adsorbed protein is reducing the surface potential $\Phi_{0}$ by contributing its own (positive) charge and thereby reducing the net surface charge that polarizes the bulk water. A maximum surface packing density of $10^{13} / \mathrm{cm}^{2}$ (and consequently a charge density of $+9 \times 10^{14}$ charges $\left./ \mathrm{cm}^{2}\right)$ is expected, given the radius of the cytochrome $c(\sim 1.5 \mathrm{~nm})$ and its charge $(+9)$ at neutral $\mathrm{pH}$. This is comparable to the density of (negative) charge found on both the silica and the supported bilayer surface and will greatly reduce the surface potential, in turn reducing the $\chi^{3}$ contribution to the SH signal. Previous
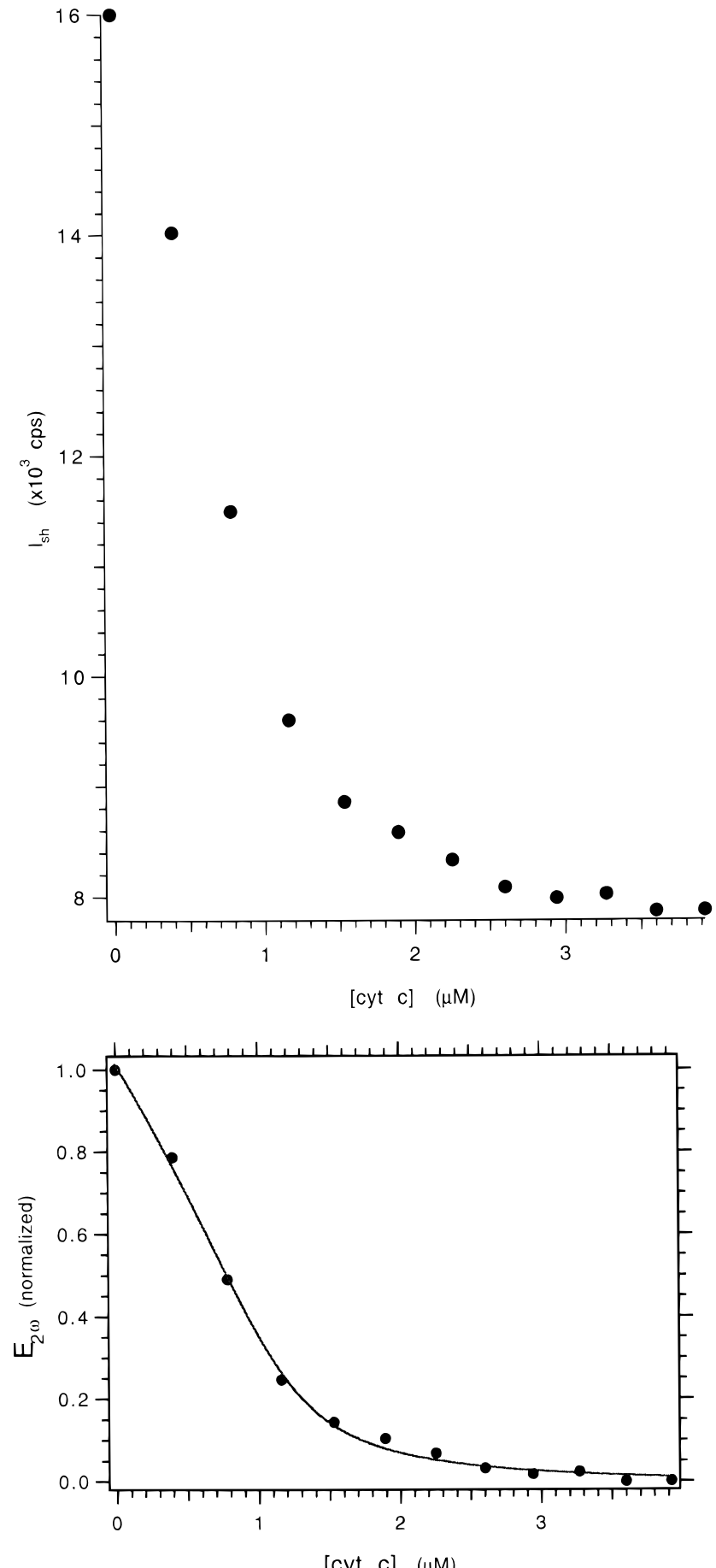

$\left[\begin{array}{lll}\text { cyt } & \mathrm{c} & (\mu \mathrm{M})\end{array}\right.$

Figure 3. SH peak intensity $\left(I_{\mathrm{SH}}\right)$ measured as a function of bulk [cyt $c]$ for the silica-water interface $(\omega=410 \mathrm{~nm})$. (b) A plot of $\left(E_{2 \omega}{ }^{3}\right.$ normalized) vs bulk [cyt $c$ ] (๑) with a fit to the Langmuir equation ${ }^{22,23}$ and using eq 2. The magnitude of the second harmonic electric field $\left(E_{2 \omega}\right)$ is proportional to the surface electric potential $\Phi_{0}$ (from eq 1). $\Phi_{0}$, in turn, is determined by the net surface charge density $\sigma=\sigma_{0}-$ $\sigma_{\text {ads }}$ with $\sigma_{0}$ the surface charge density and $\sigma_{\text {ads }}$ the charge density of the adsorbed cyt $c ; \sigma_{\text {ads }}$ is proportional to the amount of adsorbed protein which follows a Langmuir-type adsorption isotherm with bulk protein concentration.

studies show that the $\chi^{3}$ part of the signal is half that of the $\chi^{2}$ part at $\mathrm{pH} 7^{11}$ for the silica-water interface, and this is precisely the same reduction we see for saturating concentrations of cyt $c$ in our experiments with water, lending further weight to the idea that the adsorbed protein reduces the $\mathrm{SH}$ signal through the surface potential and $\chi^{3}$ effect. 
To determine how the signal changes as a function of bulk protein concentration, aliquots of protein $(\sim 1 \mu \mathrm{M})$ were added to the silica and the SH signal measured. An adsorption isotherm was obtained from this experiment, as shown in Figure 3a. The signal can be fitted using $\sigma=\sigma_{0}-\sigma_{\text {ads }}$, Equations 1 and 2, and the Langmuir equation ${ }^{20,21}$ with $\sigma$ the silica or membrane surface charge density and $\sigma_{\text {ads }}$ the charge density of the adsorbed protein. From the fit to the normalized electric field of the SH light (Figure 3a), three values were obtained: the free energy of adsorption to silica, $\Delta G_{\text {ads }}=-11.8 \mathrm{kcal} / \mathrm{mol}$, the silica surface charge density, $\sigma_{0}=-3.7 \times 10^{13}$ surface charges $/ \mathrm{cm}^{2}$, and the protein surface density at saturation, $\sigma_{\max }$ $=1.4 \times 10^{13}$ protein molecules $/ \mathrm{cm}^{2}\left(+1.3 \times 10^{14}\right.$ charges $/$ $\mathrm{cm}^{2}$ ). A similar isotherm curve was also measured in the presence of $10 \mathrm{mM}$ phosphate buffer and silica, and with the supported bilayer. A precision of one part in one hundred is readily measurable for the $\mathrm{SH}$ intensity. Given that the full $\mathrm{SH}$ reduction at saturation corresponds to a full monolayer of cyt $c$ coverage, the technique is sensitive to 0.01 monolayer of adsorbed protein or $10^{11}$ molecules $/ \mathrm{cm}^{2}$ for a close packed monolayer of $10^{13} / \mathrm{cm}^{2}$. For comparison, surface plasmon resonance requires a minimum of $50 \mathrm{pg} / \mathrm{mm}^{2}$ of protein to be detectable ${ }^{5}$ using a molecular weight of $2 \times 10^{4} \mathrm{kDa}$, this corresponds to $10 \mathrm{pmol} / \mathrm{cm}^{2}$. Detection by second harmonic generation as shown here is more sensitive than SPR by a factor of 100. No reduction in SH signal was detected when myoglobin ( $\mathrm{pI}=8.0$ ) was added, even at millimolar concentrations, further indicating that the effect is due to the electrostatic interaction between cytochrome $c$ and the surfaces.

In summary, we describe a means for detecting surfaceadsorbed protein through the effect the protein has on the polarized water molecules near the interface. In our experiments with cyt $c$ and negatively charged surfaces, the (positively charged) protein reduces the SH signal. The general applicability of the technique suggests other uses for it, for example, in studies of protein-protein binding at interfaces, especially in studies involving membrane proteins and supported lipid bilayers. Furthermore, because the technique does not require a planar surface or a TIR geometry, ${ }^{22,23}$ it could be applied to studies involving liposomes, microscopic particles, and biological cells.
Acknowledgment. The authors thank the National Science Foundation for their support.

\section{References and Notes}

(1) Lodish, H.; Darnell, J. E. Molecular Cell Biology; W. W. Freeman and Co.: New York, 1995.

(2) Tatulian, S. A. Surface Chemistry and Electrochemistry of Membranes; Dekker: New York, 1999.

(3) Gennis, R. B. Biomembranes; Springer-Verlag: New York, 1989.

(4) Biosensors in Analytical Biotechnology; Freitag, R., Ed.; Academic Press: San Diego, 1996.

(5) Earp, R. L.; Dessy, R. E. Commercial Biosensors; Ramsay, G., Ed.; Wiley: New York, 1998; Chapter 4.

(6) Schuster, S. C.; Swanson, R. V.; Alex, L. A.; Bourret, R. B.; Simon, M. I. Nature 1993, 365, 343. 6159.

(7) Brian, A. A.; McConnell, H. M. Proc. Natl. Acad. Sci. 1984, 81,

(8) Salafsky, J. S.; Groves, J. T.; Boxer, S. G. Biochemistry 1996, 35 , 14773.

(9) Eisenthal, K. B. J. Phys. Chem. 1996, 100, 12997.

(10) Corn, R. M.; Higgins, D. A. Chem. Rev. 1994, 94, 107. 327.

(11) Ong, S.; Zhao, X.; Eisenthal, K. B. Chem. Phys. Lett. 1992, 191,

(12) Zhao, X.; Ong, S.; Wang, H.; Eisenthal, K. B. Chem. Phys. Lett. 1993, 214, 203

(13) Chen, Z.; Sheves, M.; Lewis, A. Biophys. J. 1994, 67, 1155.

(14) Bard, A. J..; Faulkner, L. R. Electrochemical Methods; Wiley: New York, 1980; Chapter 12.

(15) Salafsky, J. S.; Eisenthal, K. B. Chem. Phys. Lett. 2000, 319, 435.

(16) Heimburg, T.; Marsh, D. Biophy. J. 1995, 68, 536.

(17) Pachence, J. M.; Blasie, J. K. Biophys. J. 1991, 59, 894.

(18) Kondo, A.; Higashitani, K. J. Colloid Interface. Sci. 1992, 150, 344.

(19) Chattoraj, D. K.; Birdi, K. S. Adsorption and the Gibbs Surface Excess; Plenum Press: New York, 1884.

(20) Wang, H. Thesis, Columbia University, 1996.

(21) The Langmuir equilibrium states that: $\mathrm{d} N / \mathrm{d} t=k_{1}(C-N) / 55.5$ $\left(N_{\max }-N\right)-k_{-1} N$ where $C$ is the bulk concentration of the adsorbing species, $N$ the number of adsorbed molecules per unit volume, $N_{\max }$ the maximum number adsorbed (at saturation) per unit volume, and $k_{1}$ and $k_{-1}$ are the on and off rate constants for the adsorption process. Using eq 1 , the relation $\sigma=\sigma_{0}-\sigma_{\text {ads }}$ and the solution to the Langmuir equation, $E_{2 \omega}{ }^{3}$ can be fitted as a function of $N_{\max }, K$, and $N_{0} . N_{0}$ and $N_{\max }$ can be converted to surface densities $\left(\sigma_{0}, \sigma_{\max }\right)$ using the known sample volume and prism area.

(22) Srivastava, A.; Eisenthal, K. B. Chem. Phys. Lett. 1998, 292, 345.

(23) Wang, H.; Yan, E. C. Y.; Borguet, E. Chem. Phys Lett. 1996, 259, 